\title{
Identification and antimicrobial susceptibility of microbial agents of otitis externa in dogs"
}

\author{
SERPIL KAHYA DEMIRBILEK, ÖZGE YILMAZ
}

\author{
Uludag University, Faculty of Veterinary Medicine, Department of Microbiology, 16.059, Görükle-Bursa, Turkey
}

\section{Kahya Demirbilek S., Yılmaz Ö. \\ Identification and antimicrobial susceptibility of microbial agents of otitis externa in dogs \\ Summary}

In this study, a total of 277 unmedicated dogs with otitis externa were used. Overall, 413 agents were isolated from 277 ear swab samples; $52.7 \%$ of the cases were mono-infections (146 cases), and $37.1 \%$ of the cases were poly-infections (103 cases). In $10.1 \%$ (28) of the cases, neither bacteria nor yeasts were isolated. Coagulase-positive Staphylococcus spp. were the most frequently isolated bacteria and were found in 90 $(21.8 \%)$ of the samples. Fifty-eight samples, $(\mathbf{1 4 \%})$ were positive for Staphylococcus aureus, $51(\mathbf{1 2 . 3 \% )}$ for Pseudomonas aeruginosa, 27 (6.5\%) for Proteus mirabilis, 27 (6.5\%) for Malassezia pachydermatis, 21 (5\%) for Corynebacterium spp., 21 (5\%) for $\beta$-haemolytic Streptococcus spp., 15 (3.6\%) for Staphylococcus pseudointermedius, 12 (2.9\%) for Proteus spp., 12 (2.9\%) for Escherichia coli, 9 (2.1\%) for Acinetobacter calcoaceticus, $7(1.6 \%)$ for Trichophyton mentagrophytes, $5(1.2 \%)$ for Staphylococcus auricularis, and 46 (11.1\%) for different bacteria and yeasts. A total of 14 different bacteria and yeasts were isolated and identified. Kirby-Bauer antibiotic susceptibility testing was carried out for 10 different antibiotics. The bacterial isolates were found to be resistant to amoxicillin-clavulanic acid $(\mathbf{4 5 \%})$, gentamycin $(28 \%)$, ampicillin/cloxacillin $(69 \%)$, tobramycin $(28 \%)$, amikacin $(23 \%)$, enrofloxacin $(47 \%)$, chloramphenicol $(58 \%)$, doxycycline $(65 \%)$, lincomycin/spectinomycin (58\%) and polymyxin B (62\%). In conclusion, it is important to test the antimicrobial sensitivity of aetiological agents of otitis externa before treatment so as to prevent the development of antibiotic resistance in bacteria and yeasts.

Keywords: otitis externa, dogs, bacterial isolation, yeast, antimicrobial susceptibility

Otitis externa $(\mathrm{OE})$ is defined as an acute or chronic inflammation of the epithelium of the external ear (15). The disease is more frequent in dogs than in cats. Although $\mathrm{OE}$ is not a life-threatening disease, it can be frustrating for both the patient and the owner, because of the risk of zoonotic infection and antibiotic resistance development $(1,15)$. The external ear canal is similar in structure to the interfollicular epidermis of the skin. It is a stratified cornifying epithelium with adnexal organs, such as hair follicles and their associated sebaceous and ceruminous glands. Therefore, any disease that affects the skin can also affect the external ear canals. The predisposing factors for $\mathrm{OE}$ are ear conformation, excessive moisture, obstructive ear disease, systemic disease and treatment effects (11).

The most recent classification for the aetiology of otitis externa has been proposed by Griffin (4). In this system, the aetiology of otitis externa can be divided into primary and secondary causes, which are,

${ }^{1)}$ The article is part of the research project (V-2017/36) entitled "Identification of bacteria from otitis externa in dogs" supported by the Scientific Research Fund of Uludag University. respectively, diseases or infections that directly cause inflammation in the ear, and perpetuating or predisposing factors that contribute to ear disease. The latter do not cause ear disease in their own right, but may prevent resolution of the disease and lead to recurrence if they are not dealt with adequately. Significant primary causes of otitis externa are allergy, endocrine dysfunction, autoimmune/immune-mediated disorders, keratinisation disorders, ectoparasites, foreign bodies and idiopathic causes. Significant secondary causes of otitis externa are acute bacterial disease, chronic bacterial disease and yeast infection (11).

A number of predisposing factors have been identified, such as high humidity, foreign bodies in the ear canal, obstructive diseases, immunosuppression, hair in the ear canal, hair on the concave ear surface, and pendulous ears. Other causes include progressive pathological alterations and opportunistic infection by the normal ear canal microflora, which consists of coagulase-positive and coagulase-negative Staphylococcus spp., Proteus mirabilis (P. mirabilis), Escherichia coli (E. coli), Pseudomonas aeruginosa (P. aeruginosa), 
etc. It should be remembered, however, that bacteria and yeasts are opportunists and not primary pathogens that cause only otitis externa, and they can find favourable conditions for growth provided that another cause is present (13). The condition may progress to otitis media, which serves as a source of infectious agents, resulting in chronic hyperplastic changes and obstruction of the ear canal (6).

It is well known that microbial drug resistance is a growing global problem. In developing countries, this problem is particularly acute due to the misuse of antibiotics and inadequate antibiotics. Antibiotics are used to treat infections, but most organisms acquire resistance. Reports of the continuous emergence of resistant pathogens and the changing pattern of antibiotic effectiveness in the treatment of OE necessitated this study. Therefore, antibiotic susceptibility tests were performed to evaluate treatments for chronic OE.

The aim of this study was to provide updated information on the bacterial and fungal flora involved in canine otitis externa and on the antimicrobial susceptibility patterns of some antibiotics that are most commonly used for the treatment of this disease.

\section{Material and methods}

Samples. A total of 277 dogs with otitis externa were used in this study. These dogs did not get antibiotic treatment before the ear swabs were obtained. The ear swabs were collected from the horizontal portion of the external ear canal with swabs that were placed in Stuart's transport medium and sent to the laboratory of the Microbiology Department at the Faculty of Veterinary Medicine at Uludag University within 18 hours of collection.

Bacteriological examinations. Ear swab samples were inoculated onto sheep blood agar (Columbia agar with 5\% sheep blood) and MacConkey agar (Oxoid, cat. no. CM0007R, UK) plates. Mycological tests included aerobic cultivation of the samples on Sabouraud Dextrose Agar (SDA) (Oxoid, cat. no. CM0041B, UK) for 4-14 days at $25^{\circ} \mathrm{C}$ and $37^{\circ} \mathrm{C}$. Plates were incubated at $37^{\circ} \mathrm{C}$ under aerobic conditions and examined daily for 5 days for the presence of bacterial growth. The isolated bacteria were identified on the basis of their cultural, morphological, and biochemical characteristics (14). A species was regarded as significant in causing the condition if there was a substantial growth of the species in with bacterial otitis externa a mixed culture or a moderate growth of the species in a pure culture (Clinical and Laboratory Standards Institute (CLSI) (3). Fungal species were identified by various methods, such as assessment of colony morphology, texture and surface pigmentation, microscopic examination and culture (on chrome agar). Gram staining was performed for the identification of yeasts and yeast-like fungi.

Antibiotic sensitivity test. The response of the isolates to antimicrobial drugs was tested by the disk agar diffusion method according to the CLSI guidelines (3). The 10 antimicrobial agents tested and their respective concentrations were as follows: amoxicillin-clavulanic acid $(30 \mu \mathrm{g})$, gentamycin $(120 \mu \mathrm{g})$, ampicillin/cloxacillin $(25 \mu \mathrm{g})$, tobramycin $(10 \mu \mathrm{g})$, amikacin $(30 \mu \mathrm{g})$, enrofloxacin $(5 \mu \mathrm{g})$, chloramphenicol $(30 \mu \mathrm{g})$, doxycycline $(30 \mu \mathrm{g})$, lincomycin/spectinomycin $(9 / 100 \mu \mathrm{g})$, and polymyxin B $(10 \mu \mathrm{g})$. The reference bacterial strains E. coli (ATCC 25922) and Staphylococcus aureus (S. aureus) (ATCC 25923) were used as quality control strains according to the CLSI recommendations.

\section{Results and discussion}

Bacteriological examination. The distribution of the microbial agents involved in co-infections $(100$ cases) in dogs with bacterial otitis externa by species is summarized in Table 1. A total of 413 agents were isolated from 277 ear swab samples; $52.7 \%$ of the cases were mono-infections (146 cases), and 37.1\% of the cases were poly-infections (103 cases). In $10.1 \%$ (28) of the cases, neither bacteria nor yeasts were isolated. At least two species were isolated from 103 samples: 76 samples had 2 microbial species, 24 had 3 species, and 3 had 5 species (Tab. 1). The most frequent microbial species involved in polyinfections were coagulase-positive Staphylococcus spp., $\beta$-haemolytic Streptococcus spp. (16/277, 5.7\%),

Tab. 1. Distribution of microbial agents by species involved in co-infections (100 cases) in dogs

\begin{tabular}{|c|c|c|}
\hline & Bacterial/yeast associations & $\begin{array}{l}\text { Number and } \\
\text { frequency (\%) }\end{array}$ \\
\hline \multicolumn{3}{|l|}{2 Species } \\
\hline \multirow{4}{*}{ Coagulase-positive Staphylococcus spp. } & $\beta$-haemolytic Streptococcus spp. & $16(5.7 \%)$ \\
\hline & Pseudomonas aeruginosa & $13(4.6 \%)$ \\
\hline & Escherichia coli & $9(3.2 \%)$ \\
\hline & Corynebacterium spp. & $7(2.5 \%)$ \\
\hline \multirow{2}{*}{ Pseudomonas aeruginosa } & Escherichia coli & $7(2.5 \%)$ \\
\hline & $\beta$-haemolytic Streptococcus spp. & $12(4.3 \%)$ \\
\hline \multirow{2}{*}{ B-haemolytic Streptococcus spp. } & Escherichia coli & $9(3.2 \%)$ \\
\hline & Corynebacterium spp. & $6(2.1 \%)$ \\
\hline \multirow{2}{*}{ Acinetobacter calcoaceticus } & Escherichia coli & $5(1.8 \%)$ \\
\hline & Proteus mirabilis & $4(1.4 \%)$ \\
\hline \multicolumn{3}{|l|}{3 species } \\
\hline \multirow{2}{*}{ Coagulase positive Staphylococcus spp. } & Proteus mirabilis/Escherichia coli & $4(1.4 \%)$ \\
\hline & $\beta$-haemolytic Streptococcus spp./Pseudomonas aeruginosa & $3(1 \%)$ \\
\hline B-haemolytic Streptococcus spp. & Corynebacterium spp./Acinetobacter calcoaceticus & $3(1 \%)$ \\
\hline Pseudomonas aeruginosa & 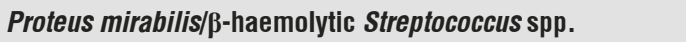 & $2(0.7 \%)$ \\
\hline
\end{tabular}


P. aeruginosa $(13 / 277,4.6 \%)$, E. coli $(9 / 277,3.2 \%)$ and Corynebacterium spp. (7/277, 2.5\%). Other species involved in co-infections are listed in Table 1. Although 3 samples were positive for 5 microbial species, these 3 samples were most probably contaminated with normal microflora or environmental microorganisms.

Coagulase-positive Staphylococcus spp. were the most frequently isolated bacteria, found in $90(21.8 \%)$ of the samples. Fifty-eight samples (14\%) were positive for $S$. aureus, $51(12.3 \%)$ for P. aeruginosa, 27 (6.5\%) for P. mirabilis, 27 (6.5\%) for Malassezia pachydermatis (M. pachydermatis), 21 (5\%) for Corynebacterium spp., 21 (5\%) for $\beta$-haemolytic Streptococcus spp., $15(3.6 \%)$ for Staphylococcus pseudointermedius, 12 (2.9\%) for Proteus spp., 12 (2.9\%) for E. coli, 9 (2.1\%) for Acinetobacter calcoaceticus (A. calcoaceticus), 7 (1.6\%) for Trichophyton mentagrophytes, 5 (1.2\%) for Staphylococcus auricularis, and 46 (11.1\%) for different bacteria and yeasts. On top of all this, we isolated and identified 5 Flavobacterium spp., 5 Mucor spp., 4 Alcaligenes spp., 4 Enterobacter spp., 4 Microsporum canis, 4 Penicillium spp., 3 Pseudomonas fluorescens, 3 Branhamella spp., 3 Providencia stuartii, 3 Penicillium spp., 2 Corynebacterium urealyticum, 2 Neisseria spp., 2 Enterococcus spp. and 2 Candida spp.

These findings were similar to those in other parts of the world, where $21.8 \%$ of all microbial isolates belonged to the coagulase-positive staphylococci (6, $7,10,12,13,15,18)$. The presence of Pseudomonas spp. and Corynebacterium spp. in dogs often indicates a chronic disease. Yeasts are commensal flora in the external ear canal of dogs that are predisposed to initial opportunistic infection by damp environmental conditions and frequent antimicrobial therapy. For example; $M$. pachydermatis is a common fungal pathogen of otitis externa in dogs; this fungus is also commensal in the ear. As is common with other types of skin infection, ear infections usually occur secondary to an underlying cause. These underlying factors can be divided into 3 categories. In reality, bacteria are secondary causes of otitis externa because infection with either yeasts or bacteria does not occur in a normal ear.

Among 257 ear swab samples from Bulgarian dogs with otitis externa, bacterial and yeast species were isolated in $93.77 \%$ of cases; the mono-infections (109 cases) involved mainly coagulase-positive staphylococci (60 strains), M. pachydermatis (97 strains) and P. aeruginosa (42 strains), and these organisms were found even more often in polyinfections (132 cases) (13). A total of 234 unmedicated dogs with otitis externa were used. A total of 18 different microbial genera (eleven bacterial genera and seven fungal genera) were identified. Two microorganisms were isolated in 26 cases, and in 84 samples, three or more agents were isolated. In addition, no bacterial and fungal growth was seen in 48 samples. Sarierler et al. (17) studied 81 clinical cases of otitis externa and isolated a Staphylococcus sp. (48.6\%), a Pseudomonas sp. $(21.5 \%)$, a Proteus sp. $(14.95 \%)$, E. coli $(8.41 \%)$ and a Klebsiella sp. (6.54\%). Mande et al. (6) examined 78 dogs with clinical signs of otitis externa, and the microbial isolates recovered were $S$. aureus (40/78, 51.3\%), Streptococcus and Pseudomonas spp. (11/78, 14.1\%), Proteus spp. (8/78, 10.2\%), E. coli $(2 / 78,2.6 \%)$, Corynebacterium spp. $(1 / 78,1.3 \%)$ and yeasts $(3 / 78,3.8 \%)$, which were infrequently isolated by Murugan et al. (8). Öztürk et al. (10) studied 58 ear swab samples from 41 dogs with otitis externa. As far as mycotic infections are concerned, Kamaljyoti et al. (5) evaluated 115 dogs with history and clinical signs of otitis externa and found 22 infections with Malassezia, 4 with Aspergillus, 3 with Candida and 1 with Cryptococcus. Nardoni et al. (9) collected auricular cerumen from 115 cats and 203 dogs and isolated M. pachydermatis in pure culture from $28.3 \%$ of cats and from $87.1 \%$ of dogs.

Antibiotic sensitivity test: The response of the bacterial isolates to antimicrobial drugs is summarized in Table 2. The isolates were resistant to amoxicillinclavulanic acid (45\%), gentamycin (28\%), ampicillin/

Tab. 2. Antibiotic resistance (\%) of microbial agents isolated from dogs with bacterial otitis externa

\begin{tabular}{|c|c|c|c|c|c|c|c|c|c|}
\hline Antibiotics & 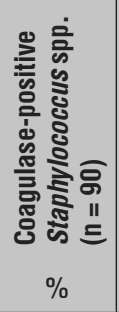 & 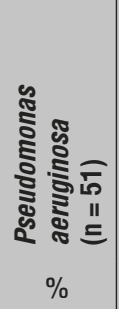 & 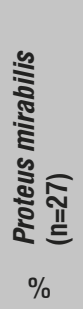 & 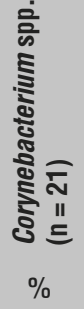 & 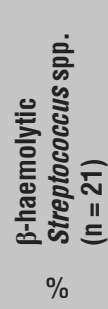 & 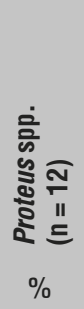 & 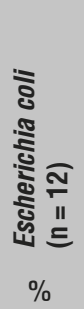 & 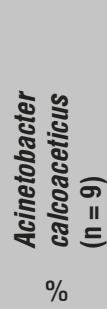 & Total \\
\hline Amoxicillin-clavulanic acid & 5 & 98 & 28 & 97 & 19 & 38 & 25 & 52 & 45 \\
\hline Gentamycin & 22 & 1 & 23 & 50 & 17 & 26 & 26 & 56 & 28 \\
\hline Ampicillin/cloxacillin & 13 & 96 & 100 & 97 & 19 & 100 & 88 & 43 & 69 \\
\hline Tobramycin & 23 & 23 & 2 & 65 & 33 & 7 & 4 & 67 & 28 \\
\hline Amikacin & 11 & 3 & 0 & 76 & 23 & 5 & 9 & 56 & 23 \\
\hline Enrofloxacin & 27 & 35 & 17 & 100 & 87 & 19 & 23 & 67 & 47 \\
\hline Chloramphenicol & 37 & 100 & 68 & 97 & 1 & 78 & 7 & 78 & 58 \\
\hline Doxycycline & 39 & 100 & 85 & 86 & 34 & 77 & 15 & 86 & 65 \\
\hline Lincomycin/spectinomycin & 63 & 36 & 66 & 89 & 46 & 67 & 8 & 93 & 58 \\
\hline Polymyxin B & 61 & 1 & 65 & 94 & 97 & 87 & 1 & 89 & 62 \\
\hline Total & 241 & 493 & 454 & 851 & 376 & 504 & 206 & 687 & \\
\hline
\end{tabular}


cloxacillin (69\%), tobramycin (28\%), amikacin (23\%), enrofloxacin $(47 \%)$, chloramphenicol $(58 \%)$, doxycycline $(65 \%)$, lincomycin/spectinomycin $(58 \%)$ and polymyxin B $(62 \%)$. The analysis of the strain response to antibiotics showed a preserved high resistance to tetracyclines $(65 \%)$, penicillins $(57 \%)$, quinolones $(47 \%)$, lincosamides $(58 \%)$ and polypeptides $(62 \%)$.

Development of antibiotic resistance is of great concern. Since the analysis of the strain response to antibiotics showed a preserved resistance to tetracyclines, penicillins and quinolones, these drugs may not be effective in treating bacterial otitis externa in dogs. Additionally, a high sensitivity to aminoglycosides was seen.

The susceptibility profiles of the bacteria provide evidence for bacterial resistance to many antimicrobial agents in the form of multiple drug resistance (MDR). This can be clearly seen from the susceptibility patterns of Corynebacterium spp. and A. calcoaceticus, as well as other bacteria, in Table 2. The results of our study showed the following average resistance values for bacteria: Corynebacterium spp. (77.3\%), A. calcoaceticus (62.4\%), Proteus spp. (45.8\%), P. aeruginosa (44.8\%), P. mirabilis (41.2\%), $\beta$-haemolytic Streptococcus spp. (34.1\%), coagulase-positive Staphylococcus spp. (21.9\%), and E. coli $(18.7 \%)$. These values differ from those obtained in other studies $(13,15,16)$, and this difference in the susceptibility profiles might be due to the different frequency of use of these agents in different geographic locations for the treatment of ear infections.

In conclusion, the isolation and identification of microorganisms are important for the correct diagnosis and correct treatment of otitis externa in dogs. The bacterial agents most commonly isolated from canine otitis externa were coagulase-positive staphylococci. It should be remembered, however, that yeasts are also frequently involved in the aetiology of ear canal inflammations, and further research is required to determine the importance of yeasts in the pathogenesis of otitis externa. All data from this study showed that further research is required to determine the importance of antibiotics in the treatment of otitis externa in dogs. These results highlight the need to combine antimicrobial susceptibility tests with bacterial isolation. In addition, susceptibility data would be useful in determining the most suitable approaches/strategies for medical management.

\section{References}

1. August J. R.: Otitis externa. A disease of multifactorial etiology. Vet. Clin. North Am. Small Anim. Pract. 1988, 18, 731-742.

2. Borum A. E., Çeçen G., Demir G., Çetin C., Şentürk S.: Determination and antimicrobial susceptibility of microorganisms isolated from otitis externa with dogs. Kocatepe Vet. Derg. 2014, 7, 27-31.

3. CLSI (Clinical Laboratory Standard Institute): National committee for clinical and laboratory standards and methods for antimicrobial susceptibility testing of anaerobic bacteria, Approved standard. $6^{\text {th }}$ edition, NCCLS, Wayne, PA, Document M11-A6, 2004.
4. Griffin C. E.: Classifying cases of otitis externa the PPSP System. Pro. ESVD Workshop on Otitis. St Helens 2010

5. Kamaljyoti A. K. S., Sood N. K., Sharma S., Filia G.: Epidemiology and diagnosis of mycotic infections in canine otitis externa. Intas Polivet 2016 17, 302-303.

6. Mande J. D., Kitaa J. M. A.: Microbial profile and antimicrobial susceptibility of isolates from dogs with otitis externa in Kenya. J. Kenya Vet. Assoc. 2005, 29, 40-44.

7. Moraes L. A., Pereira J. M. M., Silva S. P., Moreira V. M. T., Casseb A. Microbiological diagnosis and bacterial multiresistance in vitro of external otitis externa of dogs. Veterinaria Zootecnia 2014, 21, 98-101.

8. Murugan M. S., Parthiban S., Malmarugan S., Rajeshwar J. J.: Antibiogram and therapeutic management of bacterial otitis externa: A clinical study of 81 dogs. Intas Polivet 2016, 17, 292-294.

9. Nardoni S., Ebani V. V., Fratini F., Mannella R., Pinferi G., Mancianti F., Finotello R., Perrucci S.: Malessezia, mites and bacteria in the external ear canal of dogs and cats with otitis externa. Slov. Vet. Res. 2014, 51, 113-118.

10. Öztürk D., Pehlivanoğlu F., Türütoğlu H., Şirin Y. S., Şababoğlu E.: Otitis externalı köpeklerden izole edilen mikroorganizmalar ve antibiyotik duyarlıl1kları. Euro. J. Vet. Sci. 2016, 32, 84-88.

11. Paterson S.: Discovering the causes of otitis externa. In Practice, Focus 2016 http://dx.doi.org/10.1136/inp.i470.

12. Perry L. R., Maclennan B., Korven R., Rawlings T. A.: Epidemiological study of dogs with otitis externa in Cape Breton, Nova Scotia. Can. Vet. J. 58, 168-174 .

13. Petrov V., Mihaylov G., Tsachev I., Zhelev G., Marutsov P., Koev K.: Otitis externa in dogs: microbiology and antimicrobial susceptibility. Rev. Med. Vet. 2013, 164, 18-22.

14. Quinn P. J., Carter M. E., Markey B.: Section: 2 Bacteriology, [in:] Clinical Veterinary Microbiology. Harcourt Publishers Limited, USA 1999, p. 118-254.

15. Rosser E. J.: Causes of otitis externa. Vet. Clinics Small Animal. 2004, 34, 459-468.

16. Rosychuk R. A. W.: Management of otitis externa. Vet. Clin. North Am. Small Anim. Pract. 1994, 24, 921-952.

17. Sarlerler M., Kırkan Ş.: Microbial diagnosis and therapy of canine otitis externa. Veteriner Cerrahi Dergisi 2004, 10, 11-15.

18. Selvakumar S., Mohamed A. M. G., Pothiappan P.: A clinical study of otitis externa with bacterial isolation, antimicrobial susceptibility and therapeutic management in 44 dogs. Intas Polivet 16, 332-333.

19. Scott D. W., Miller W. H., Griffin C. E.: Otitis, [in:] Muller and Kirk's Small Animal Dermatology. Saunders, Philadelphia 2001, p. 143-148.

Corresponding author: Assoc. Prof. Serpil Kahya Demirbilek, Uludag University, Faculty of Veterinary Medicine, Department of Microbiology, 16.000, Görükle-Bursa, Turkey; e-mail: serpilkahya@uludag.edu.tr 